\title{
La préhistoire de l'APAD
}

Jean-Pierre Olivier de Sardan

\section{(2) OpenEdition}

Journals

Édition électronique

URL : http://journals.openedition.org/apad/324

DOI : 10.4000/apad.324

ISSN : 1950-6929

Éditeur

LIT Verlag

Édition imprimée

Date de publication : 15 mars 1991

\section{Référence électronique}

Jean-Pierre Olivier de Sardan, «La préhistoire de l'APAD », Bulletin de l'APAD [En ligne], 1 | 1991, mis en ligne le 23 juin 2006, consulté le 24 septembre 2020. URL : http://journals.openedition.org/apad/324 ; DOI : https://doi.org/10.4000/apad.324

Ce document a été généré automatiquement le 24 septembre 2020.

Bulletin de I'APAD 


\title{
La préhistoire de l'APAD
}

\author{
Jean-Pierre Olivier de Sardan
}

1 Regrouper en un "réseau" ou une "association" les chercheurs africanistes s'inspirant de l' anthropologie (au sens large et dans l'acception la moins corporatiste possible) et concernés par les problèmes de changement social et de développement : l'idée initiale a germé au printemps 1990, entre Montpellier et Marseille. Cette idée devait être dans l'air, puisque d'emblée les réactions des premiers collègues consultés furent positives. Il fut donc décidé de mettre la main à la pâte. L'étonnant semblait même qu'une telle initiative n'ait pas vu le jour plus tôt. Car, après tout, cela faisait bien des années que, dans le paysage de la recherche africaniste, tous ceux qui naviguaient entre l' anthropologie, la sociologie et le développement se sentaient quelque chose en commun, et n'avaient pas de lieu pour en débattre.

2 D'emblée l'orientation fondatrice fut claire: nous entendions combiner une anthropologie fondamentaliste prenant le "développement" comme objet d'étude, et une anthropologie "appliquée" associée à des actions de "développement". Nous savions qu'il pouvait y avoir contradiction entre ces deux pôles, que les uns tireraient vers le premier et les autres vers le second, que la fonction "pratique" serait refusée par certains, et que la fonction théorique en effaroucherait d'autres. Mais c'était, selon nous, une tension nécessaire, et qui pouvait être productive. Nous le pensons toujours, et l'APAD est en quelque sorte fondée sur cette tension-là.

3 Pour le reste beaucoup de choses ont changé depuis une année. Et pas seulement parce que, depuis la dizaine de personnes qui fut vite associée au projet APAD, nous nous sommes trouvés à notre grande surprise plus de 80 à assister en tout ou en partie aux deux journées fondatrices des 5-6 mars 91, et que, aujourd'hui, plus de 200 personnes ont manifesté leur intérêt, tout cela ayant pourtant avancé sans "mailing" sérieux ni publicité aucune, en contactant les gens de bric et de broc (mille excuses pour tous ceux que nous avons oubliés...), dans un grand amateurisme fait d'improvisations et d'idées en vrac...

4 Le plus décisif n'est pas là : c'est le processus d'élargissement «qualitatif » qui a sans doute été le plus important. D'un petit noyau méridional, puis ensuite français, nous en sommes vite venus à cette conception originale, et décisive, d'une association 
"euro-africaine". Il y avait eu bien sûr des expériences antérieures pavant le chemin : des rencontres entre chercheurs européens et africains à Braga, Bologne, Giessen, des collaborations individuelles, des colloques, des échanges entre Universités et Centres de recherche, des tentatives aussi de dialogues entre chercheurs et opérateurs de développement au-delà des frontières nationales. Le premier succès de l'APAD a été de réussir à donner, malgré une massive présence française due à cette préhistoire, une réelle dimension "euro-africaine" à ses journées fondatrices. Et la première tache de l'APAD sera de développer le plus possible et aussi vite que possible cette dimension, dans le temps désormais de son histoire propre. Une présence effective et des activités de l'APAD en Afrique même en seront le signe le plus manifeste.

Un autre élargissement est en chantier, et semble déjà acquis au moins dans les têtes : l'ouverture de l'APAD aux "non-anthropologues". C'était évident dès le départ en ce qui concerne les autres sciences sociales: après tout les sociologues africanistes sont presque tous anthropologues aussi, et nombreux sont les économistes, les géographes, les politologues à utiliser une démarche d'inspiration anthropologique. Mais l'élargissement commence à s'opérer vers d'autres partenaires: des chercheurs de disciplines "techniques" (médecins, agronomes, hydrauliciens...), et des opérateurs de développement (formateurs, chefs de projets, experts...). Autrement dit la référence à l'anthropologie dans l'intitulé de l' APAD doit être interprétée comme un souci commun de tous ses adhérents, quelle que soit leurs "spécialités", de porter attention aux représentations et aux logiques sociales de l'ensemble des acteurs sociaux concernés par le changement social et le développement: ceci implique certaines formes d'attention, d'écoute, d'analyse, de compétence, qui relèvent d'une démarche anthropologique, sans être le monopole des anthropologues loin de là.

Une dernière précision sur l'expression "changement social et développement. Aussi "classique" et vague que soit le terme de "changement social", il nous a finalement semblé indispensable qu'il précède le terme "développement" (dont nous avons une conception essentiellement non normative: il y a "développement" là où des institutions de développement mènent des actions de développement...) : il fallait en effet signifier que d'une part le "développement" n'est qu'une forme historique particulière $d u$ changement social, et que, d'autre part, nous entendions nous intéresser aussi aux processus de transformation et aux dynamiques sociales intervenant "hors développement".

7 Voilà pour la pré-histoire de l'APAD, appréhendée non au niveau anecdotique, mais par rapport à son contenu et à sa dynamique intellectuelle. Ce "Bulletin de "APAD" n ${ }^{\circ} 1$ en porte témoignage et constitue en quelque sorte l'acte de naissance de notre association.

Présentons-le "sommairement". Vous pourrez lire tout d'abord le "texte d'orientation" qui a servi pendant un an de référence pour constituer le réseau de départ : "Pourquoi une Association euro-africaine pour l'anthropologie du changement social et du développement ?". Remanié peu à peu, amélioré par les uns et les autres, il définit bien nos motifs et nos objectifs. C'est une plate-forme de départ.

9 Vous trouverez ensuite une série de documents issus des deux journées fondatrices des 5 et 6 mars 1991 à Paris, et qui se distribuent entre les diverses rubriques, désormais régulières, de ce Bulletin.

10 La rubrique "Réflexions et débats" est alimentée par ce qui constitua la première journée : des exposés visant il susciter des discussions. Il s'agit d'autant de "points de vue", parfois plus théoriques, parfois plus polémiques, parfois plus didactiques. Il fallait 
mettre le débat au centre de nos activités et brasser, même dans le désordre, les idées des uns ou des autres.

11 Dans la rubrique "Vie de l'APAD" sont inclus les rapports des ateliers thématiques qui se sont réunis durant la seconde journée. Il s'agissait de lancer des "groupes de travail", si possible destinés à se pérenniser, prenant en charge une fonction d'animation intellectuelle entre personnes concernées par de mêmes préoccupations. La rubrique se termine sur un tableau récapitulatif des actions prioritaires de l'APAD pour 1991-1992.

12 La rubrique "Euro-afrique" fait une sorte d'état des lieux par pays des rapports entre anthropologie et développement. Ces présentations sont issues d'une table ronde tenue elle aussi pendant ces deux journées.

13 Mais ce numéro ne veut pas se réduire à n'être que des sortes d'"Actes" de ces journées. D'où le lancement d'autres rubriques : "Agenda", "Lu et à lire", "Courrier".

14 Faut-il vraiment insister sur le rôle que les membres de l'APAD et les lecteurs du Bulletin peuvent jouer en envoyant pour le prochain numéro textes, annonces, présentations, compte-rendus, articles, etc...?

15 Mais au fait, quand sortira le prochain Bulletin? 